\title{
Enhancement of tendon-bone healing via the combination of biodegradable collagen-loaded nanofibrous membranes and a three-dimensional printed bone-anchoring bolt
}

\author{
This article was published in the following Dove Press journal: \\ International Journal of Nanomedicine \\ 25 August 2016 \\ Number of times this article has been viewed
}

\author{
Ying-Chao Chou',2 \\ Wen-Lin Yeh² \\ Chien-Lin Chao' \\ Yung-Heng $\mathrm{Hsu}^{1,2}$ \\ Yi-Hsun $\mathrm{Yu}^{1,2}$ \\ Jan-Kan Chen ${ }^{3}$ \\ Shih-Jung Liu' ${ }^{1,2}$ \\ 'Department of Mechanical \\ Engineering, Chang Gung University, \\ ${ }^{2}$ Department of Orthopedic Surgery, \\ Chang Gung Memorial Hospital, \\ ${ }^{3}$ Department of Physiology and \\ Pharmacology, Chang Gung University, \\ Taoyuan, Taiwan
}

\begin{abstract}
A composite biodegradable polymeric model was developed to enhance tendon graft healing. This model included a biodegradable polylactide (PLA) bolt as the bone anchor and a poly(D,L-lactide-co-glycolide) (PLGA) nanofibrous membrane embedded with collagen as a biomimic patch to promote tendon-bone interface integration. Degradation rate and compressive strength of the PLA bolt were measured after immersion in a buffer solution for 3 months. In vitro biochemical characteristics and the nanofibrous matrix were assessed using a water contact angle analyzer, $\mathrm{pH}$ meter, and tetrazolium reduction assay. In vivo efficacies of PLGA/ collagen nanofibers and PLA bolts for tendon-bone healing were investigated on a rabbit bone tunnel model with histological and tendon pullout tests. The PLGA/collagen-blended nanofibrous membrane was a hydrophilic, stable, and biocompatible scaffold. The PLA bolt was durable for tendon-bone anchoring. Histology showed adequate biocompatibility of the PLA bolt on a medial cortex with progressive bone ingrowth and without tissue overreaction. PLGA nanofibers within the bone tunnel also decreased the tunnel enlargement phenomenon and enhanced tendon-bone integration. Composite polymers of the PLA bolt and PLGA/collagen nanofibrous membrane can effectively promote outcomes of tendon reconstruction in a rabbit model. The composite biodegradable polymeric system may be useful in humans for tendon reconstruction.

Keywords: polylactide-polyglycolide nanofibers, PLGA, collagen, 3D printing, polylactide, PLA, bone-anchoring bolts, tendon healing
\end{abstract}

\section{Introduction}

Rupture of the anterior and/or posterior cruciate ligament is a common knee injury sustained by athletes in high-risk sports and victims of high-speed traffic accidents. ${ }^{1}$ Successful ligament reconstruction involves penetrating a tendon graft through the bone tunnel and anchoring it between the femur and tibia. ${ }^{2}$ However, the healing rate of the tendon in the bone tunnel is relatively slow due to the lack of a suitable biointerface between the tendon graft and bone tunnel for efficient integration. ${ }^{3,4}$ Orthopedic basic science research strategies aiming to augment tendon-bone healing include the use of osteoinductive growth factors, platelet-rich plasma, and enveloping the grafts with periosteum or osteoconductive materials. ${ }^{5-9}$ In addition, numerous studies have clinically demonstrated the promotion of tendon-bone anchoring by fixation with a biodegradable interference screw or press-fit bone plug. ${ }^{10,11}$

In recent decades, polymeric biomaterials for clinical applications have received increasing attention owing to their additional potential advantages. ${ }^{12,13}$ For example,
Correspondence: Shih-Jung Liu Department of Mechanical Engineering, Chang Gung University, 259 Wen-Hwa First Road, Kwei-Shan, Taoyuan 3,

Taiwan

Tel $+886321 \mid 8166$

Fax +88632118558

Email shihjung@mail.cgu.edu.tw BY NC and incorporate the Creative Commons Attribution - Non Commercial (unported, v3.0) License (http:///creativecommons.org/licenses/lby-nc/3.0/). By accessing the work you
hereby accept the Terms. Non-commercial uses of the work are permitted without any further permission from Dove Medical Press Limited, provided the work is properly attributed. For permission hereby accept the Terms. Non-commercial uses of the work are permitted without any further permission from Dove Medie
for commercial use of this work, please see paragraphs 4.2 and 5 of our Terms (https://www.dovepress.com/terms.php). 
polylactide (PLA) is a biodegradable polymer with good mechanical strength and has been considered an ideal biomaterial for load-bearing applications, such as fracture fixation devices. ${ }^{14,15}$ Other polymeric biomaterials exhibit sustainable drug-eluting capacities that are beneficial in orthopedic surgeries, including controlled delivery of growth factors for promoting bone healing, sustainable elution of antibiotics for combating infection, and biomimetic patches for tendon or ligament reconstructions. ${ }^{16-18}$ Poly(D,L-lactide-co-glycolide) (PLGA) belongs to the class of synthesized copolymers from which absorbable sutures, absorbable surgical clips, and controlled release implants are made.

This study designed and developed a tendon fixation model based on polymeric biomaterials that provided adequate tendon-bone anchoring and enhanced tendonbone healing. The three-dimensional (3D) printing technique is a novel process by which objects to be printed out can be of almost any shape or geometry. ${ }^{19}$ On the other hand, electrospinning technique does not require the use of coagulation chemistry or high temperatures to produce solid threads from solution. All these make the processes suited to the production of fibers from molecules of biomaterials. ${ }^{20}$

A

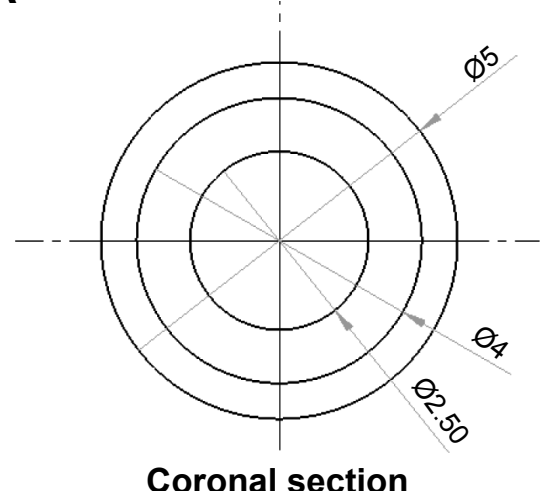

C

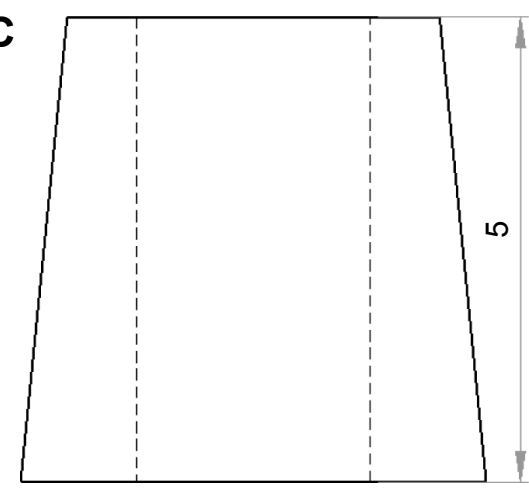

Frontal section
We hypothesized that successful tendon reconstruction can be effectively achieved using the $3 \mathrm{D}$ printing technique to fabricate a biodegradable PLA bone bolt for tendon-bone anchoring and the electrospinning technique to produce PLGA nanofibrous membranes embedded with collagen for promoting tendon-bone healing. We assessed the in vitro characteristics of the PLA bolt and PLGA membrane for their cytocompatibility. In addition, in vivo evaluations of these composite polymers for promoting tendonbone healing were conducted by wrapping a nanofibrous membrane on a tendon graft and implanting the tendon in a rabbit model.

\section{Materials and methods 3D printed PLA bolt}

\section{Preparation of the 3D printed PLA bolt}

Commercially available PLA filaments (Prolink Microsystems Corp., Taipei, Taiwan) with a diameter of $3 \mathrm{~mm}$ were used for the $3 \mathrm{D}$ printing. The bone-anchoring bolts were prepared by fused deposition modeling using a 3D printer (U-Maker, Taipei, Taiwan) with a resolution of $200 \mu \mathrm{m}$. Figure 1 shows the layout and dimensions of the truncated

\section{B}

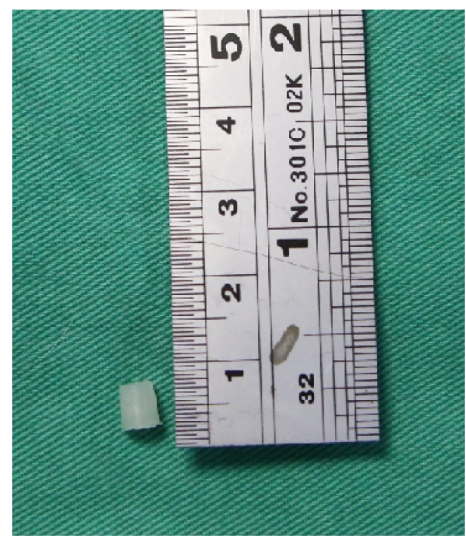

D

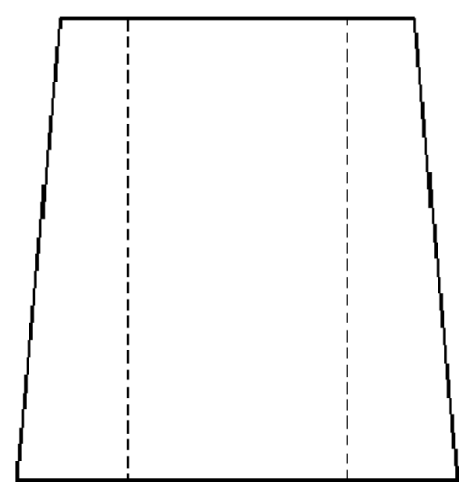

Sagital section

Figure I 3D printed PLA bone bolt.

Notes: Layout and dimensions of the truncated cone-shaped bone bolt. (A) Coronal section. (B) Photograph of the 3D printed bolt. (C) Frontal section. (D) Sagital section. Unit of measure $=\mathrm{mm}$

Abbreviations: PLA, polylactide; 3D, three dimensional. 
cone-shaped bone bolt design. During the process, the printer extruded small beads of material that harden immediately to form layers. The PLA filament that was wrapped on a coil was unwound to supply material to the extrusion nozzle head. The nozzle head heated the material and turned the flow on and off. Two stepper motors were employed to move the extrusion head and adjust the flow. A computer-aided manufacturing software package was used to develop a code that was sent to a microcontroller controlling the motors.

\section{Characterization of 3D printed bolts}

The molecular weight variation of the $3 \mathrm{D}$ printed PLA bolts was monitored by immersing them in a phosphate buffer, $0.15 \mathrm{~mol} / \mathrm{L}$ ( $\mathrm{pH} 7.4$ ), at $37^{\circ} \mathrm{C}$. The PLA samples were taken out of the buffered solution after 3 months. After drying the PLA bolts in an oven for 24 hours, their molecular weights were measured by a gel permeation chromatograph equipped with a Waters 2414 refractive index detector (Waters Corp., Milford, MA, USA).

\section{PLGA/collagen nanofibrous membranes}

Preparation of collagen-embedded nanofibrous membranes

Collagen-embedded nanofibrous membranes were fabricated by electrospinning of commercially available PLGA (Resomer RG 503; Sigma-Aldrich Co., St Louis, MO, USA), which is a biodegradable polymer blended with collagens. PLGA has a lactide:glycolide ratio of 50:50 and a molecular weight of 33,000 Da. Type I collagen from bovine achilles tendon and 1,1,1,3,3,3-hexafluoro-2-propanol as the solvent were purchased from Sigma-Aldrich Co. (St Louis, MO, USA).

The electrospinning system consisted of a syringe and a needle (internal diameter: $0.42 \mathrm{~mm}$ ), a ground electrode, an aluminum sheet, and a high voltage supply. The needle was connected to the high voltage supply, which could generate positive DC voltages and currents up to $35 \mathrm{kV}$ and $4.16 \mathrm{~mA} / 125 \mathrm{~W}$, respectively. For the electrospinning of PLGA/collagen fibers, PLGA and collagen were first dissolved in 1,1,1,3,3,3-hexafluoro-2-propanol at concentrations of $15 \%$ and $8 \%(\mathrm{w} / \mathrm{v})$, respectively. A series of PLGA/ collagen blend solutions (PLGA solution/collagen solution $=40 / 60 \mathrm{v} / \mathrm{v}, 50 / 50 \mathrm{v} / \mathrm{v}, 67 / 33 \mathrm{v} / \mathrm{v}, 80 / 20 \mathrm{v} / \mathrm{v}, 100 / 0 \mathrm{v} / \mathrm{v}$ ) were prepared by mixing each solution at predetermined ratios and then delivering them by a syringe pump at a volumetric flow rate of $4 \mathrm{~mL} / \mathrm{h}$. The distance between the needle tip and ground electrode was $15 \mathrm{~cm}$, and a positive voltage of $17 \mathrm{kV}$ was applied to the polymer solutions. All electrospinning experiments were performed at room temperature.

\section{Scanning electron microscopic observation of} nanofibers

The morphology of the electrospun PLGA/collagen nanofibers after gold coating was observed on a scanning electron microscope (SEM, Hitachi S-3000N; Hitachi Ltd., Tokyo, Japan). The average diameter and diameter distribution were obtained by measuring the diameters of 50 randomly selected fibers.

\section{Water contact angle and $\mathrm{pH}$ value measurement of} nanofibers

The water contact angles of the nanofibrous PLGA/collagen membranes were measured using a water contact angle analyzer (First Ten Angstroms, Portsmouth, VA, USA). Samples of dimensions $1 \times 1 \mathrm{~cm}$ were cut out from the membranes and placed on the testing plate after distilled water was carefully dropped on their surfaces. The contact angles were measured using a video monitor. The contact angles of the membranes with various PLGA/collagen ratios were measured. Contact angles from $0^{\circ}$ to $30^{\circ}$ were defined as highly hydrophilic, $30^{\circ}$ to $90^{\circ}$ as hydrophilic, $90^{\circ}$ to $150^{\circ}$ as hydrophobic, and $150^{\circ}$ to $180^{\circ}$ as highly hydrophobic.

The $\mathrm{pH}$ values of the eluted solutions from the PLGA/ collagen membranes on days $1,2,3,7,14,21,28,56$, and 84 were monitored by a $\mathrm{pH}$ meter (EuTech Model PH1100; Eutech Instruments Pte Ltd, Singapore).

\section{Cytotoxicity of electrospun nanofibers}

The cytotoxicity of the electrospun nanofibers was examined by the 3-(4,5-dimethylthiazol-2-yl)-2,5-diphenyltetrazolium bromide (MTT) reduction assay (Hoffman-La Roche Ltd., Basel, Switzerland) of cell viability. The electrospun PLGA/ collagen nanofibers were cut out with a punch and placed in 24-well culture plates. Human fibroblasts obtained from the foreskins of patients (1-3 years of age) undergoing surgery were seeded $\left(5 \times 10^{3}\right.$ cells/well) in Dulbecco's Modified Eagle's Medium (DMEM) at $37^{\circ} \mathrm{C}$ under a $5 \% \mathrm{CO}_{2} / 95 \%$ air condition until cell confluence. Cell viability was monitored on the first and third days by MTT assays and was quantified using an enzyme-linked immunosorbent assay reader. DMEM was set as a control group for comparison with DMEM embedded with nanofibers having five different PLGA/collagen ratios. The optical density (OD) was measured under a light source of $490 \mathrm{~nm}$.

\section{Surgical procedure and in vivo assessments} Surgical procedure and animal care

A total of 48 adult New Zealand white rabbits (Animal Health Research Institute, Panchiao, Taiwan) with a mean weight 
of $3.3 \pm 0.7 \mathrm{~kg}$ were used in this study. Under the supervision of a licensed veterinarian, all rabbits involved in this study were cared for in a manner that was consistent with the regulations of the National Institute of Health of Taiwan. All animal-related procedures received institutional approval from Chang Gung University.

During the procedure, all 48 rabbits received general anesthesia through inhalation of isoflurane (Aesica Queenborough Ltd., Queenborough, UK) in an anesthesia chamber that comprised a $40 \times 20 \times 28 \mathrm{~cm}$ transparent acrylic box. After the anesthesia induction procedure, all rabbits were carried out of the chamber and were supplied tolerable isoflurane through a mask to maintain sufficient anesthesia throughout the surgical procedure. After an aseptic preparation, all rabbits underwent a longitudinal incision and a lateral parapatellar approach to expose the left knee joint. The long digital extensor tendon was identified and detached from its insertion on the lateral femoral condyle by a sharp dissection. The free tendon tip was sutured with four bands of 3-0 Vicryl sutures (Ethicon, Somerville, NJ, USA) for further tendon procedures. The soft tissue and fascia over the tibialis anterior muscle were retracted to expose the proximal tibia. A $2.0 \mathrm{~mm}$ drill hole was created on the metaphysis of the proximal tibia perpendicular to the long axis of the bone. The length of the tunnel was measured with a depth gage. After adequate preparation of the bone tunnel, the free end of the tendon was manually pulled through the drill hole (Figure 2A). Then, the tendon tip was passed through the central hole of the 3D printed bolt and was fixed to the bolt by tendon looping and suturing with prepared bands of 3-0 Vicryl sutures (Figure $2 \mathrm{~B}$ and $\mathrm{C}$ ). The rabbits were randomly divided into two groups. In group A, 24 rabbits underwent only the aforementioned tendon transfer procedure. In group B, a nanofibrous membrane of dimensions $10.0 \times 5.0 \mathrm{~mm}$ with a PLGA/ collagen ratio of $67 / 33$ was prepared and wrapped around the free tendon end for all 24 rabbits before completing the same tendon transfer steps (Figure 3A and B).

After completing the surgical procedure, all rabbits were allowed to live freely and were given standard rabbit chow and sterilized drinking water ad libitum. The temperature and humidity in our institutional animal care center were maintained at $\sim 24^{\circ} \mathrm{C}$ and $70 \%$, respectively.

\section{Animal euthanasia for biomechanical and histological studies}

After completing the scheduled postoperative course, 24 (12 of group A, 12 of group B) rabbits were euthanized by intravenous injections of $10 \mathrm{cc}$ lidocaine at postoperative 8 weeks, and the other 24 (12 of group A, 12 of group B) rabbits were sacrificed at postoperative 16 weeks for interfacial strength tests and histological study.

At each period, four tibias with affixed transferred tendon specimens from each group were harvested for histological examination. The proximal tibia specimens were immersed in $5 \%$ formic acid for decalcification for 3 days, dehydrated in a gradient ethanol series, embedded in paraffin, sectioned at
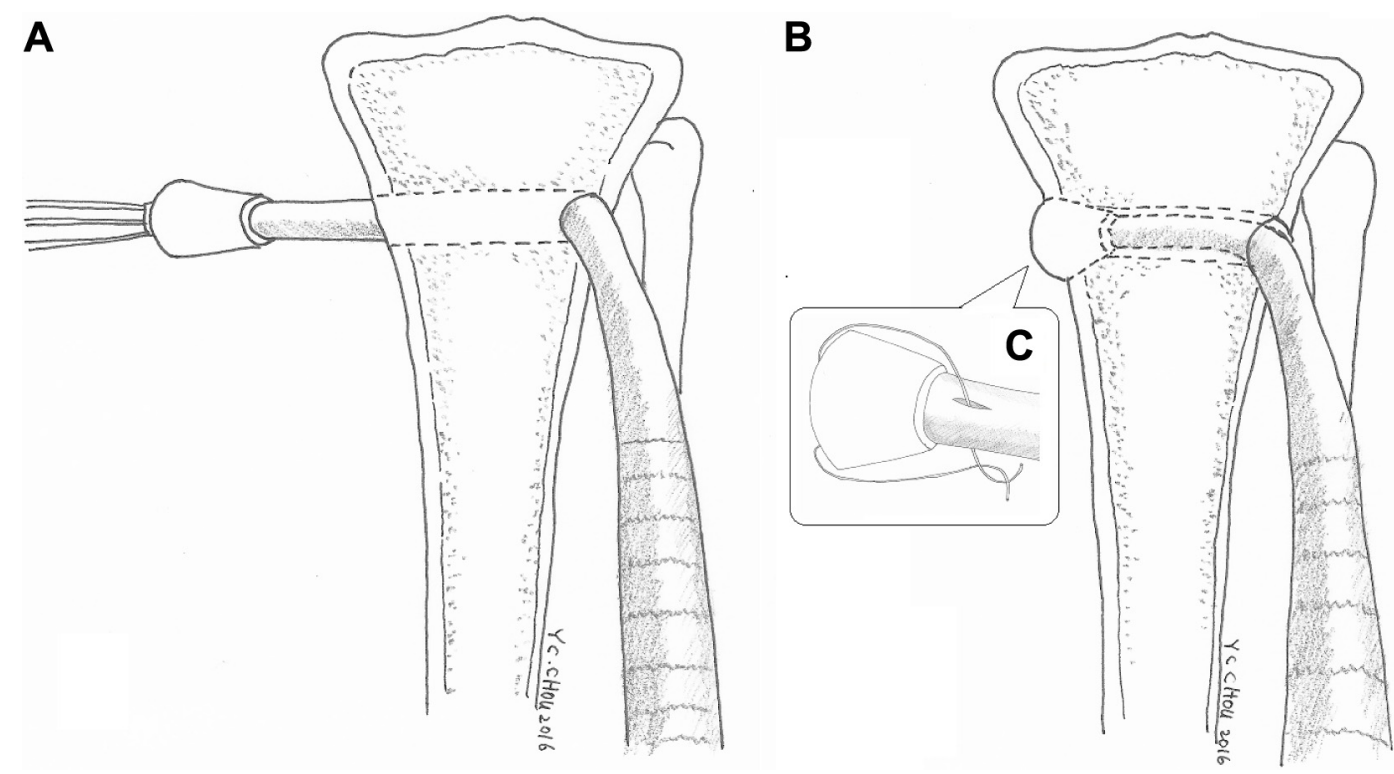

Figure 2 Illustration of a tibia model in group A (comparison group without nanofibers) that received the 3D printed anchoring bolt only.

Notes: (A) The extensor tendon penetrated through a bone tunnel on the metaphysis of the proximal tibia. (B) Final pullback of the tendon to allow bolt entrapment on the bone tunnel. (C) The tendon was fixed to the bolt by looping and suturing with prepared bands of 3-0 Vicryl sutures.

Abbreviation: 3D, three dimensional. 

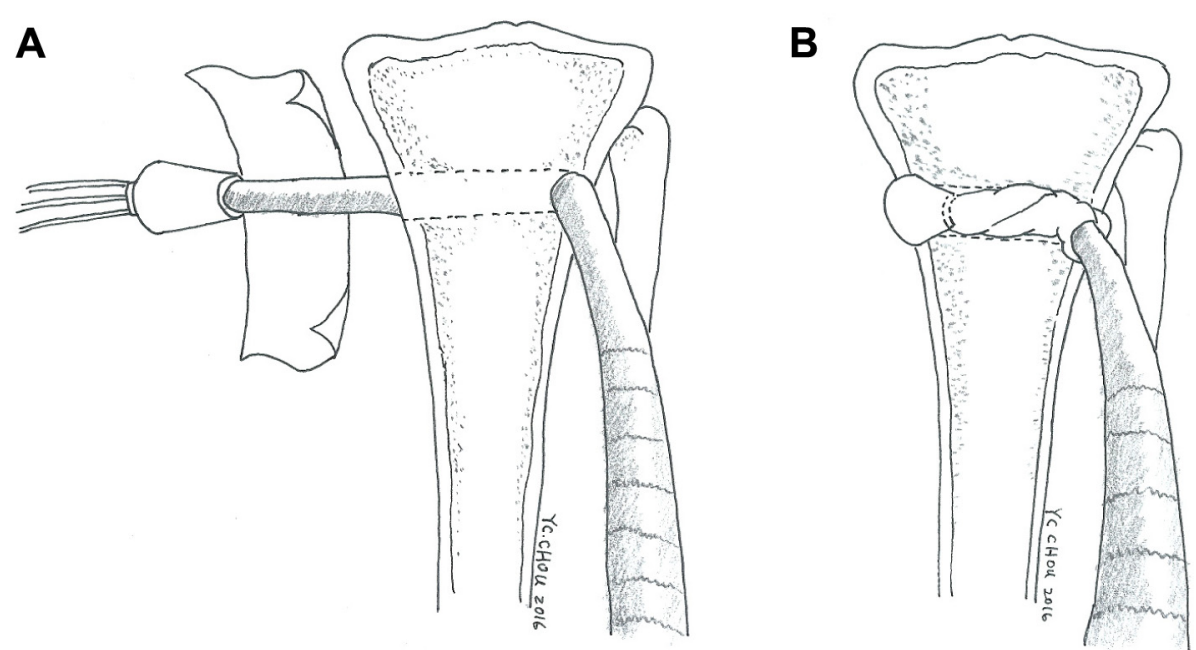

Figure 3 Illustration of a tibia model in group B (study group with nanofibers) that received the 3D printed anchoring bolt and a nanofibrous membrane wrapping around the tendon graft.

Notes: (A) The nanofibrous membrane prepared for wrapping around the tendon graft. (B) The final configuration of composite polymers used to wrap the tendon with nanofibers and anchor into the bone tunnel by the bolt.

Abbreviation: 3D, three dimensional.

thickness of $5 \mu \mathrm{m}$, and stained with hematoxylin and eosin. The medial cortical tunnel of the bolt-entrapped site and the lateral cortical tunnel of the tendon-bone interface were prepared for each tibia specimen for different histological assessments.

At each period, the other eight tibia specimens from each group were prepared for interfacial strength measurements in the healed tendon/bone test. The long digital extensor muscle and tendon were sutured using a No 5 Ethibond suture. The tibia with an affixed tendon was clamped in a specially designed fixture and then tested using a material testing system machine (MTS systems, Eden Prairie, MN, USA; Figure 4A and B). During the measurement, the tendon was pulled by the top clamp at a rate of $45 \mathrm{~mm} / \mathrm{min}$ through a distance of $10 \mathrm{~cm}$ before the clamp was returned to its starting point. The failed mode and maximum failure load were recorded for all tibia specimens.

\section{Statistics and data analysis}

Data were collected from quadruplicate samples and analyzed by one-way analysis of variance. Differences were considered statistically significant for $P<0.05$.
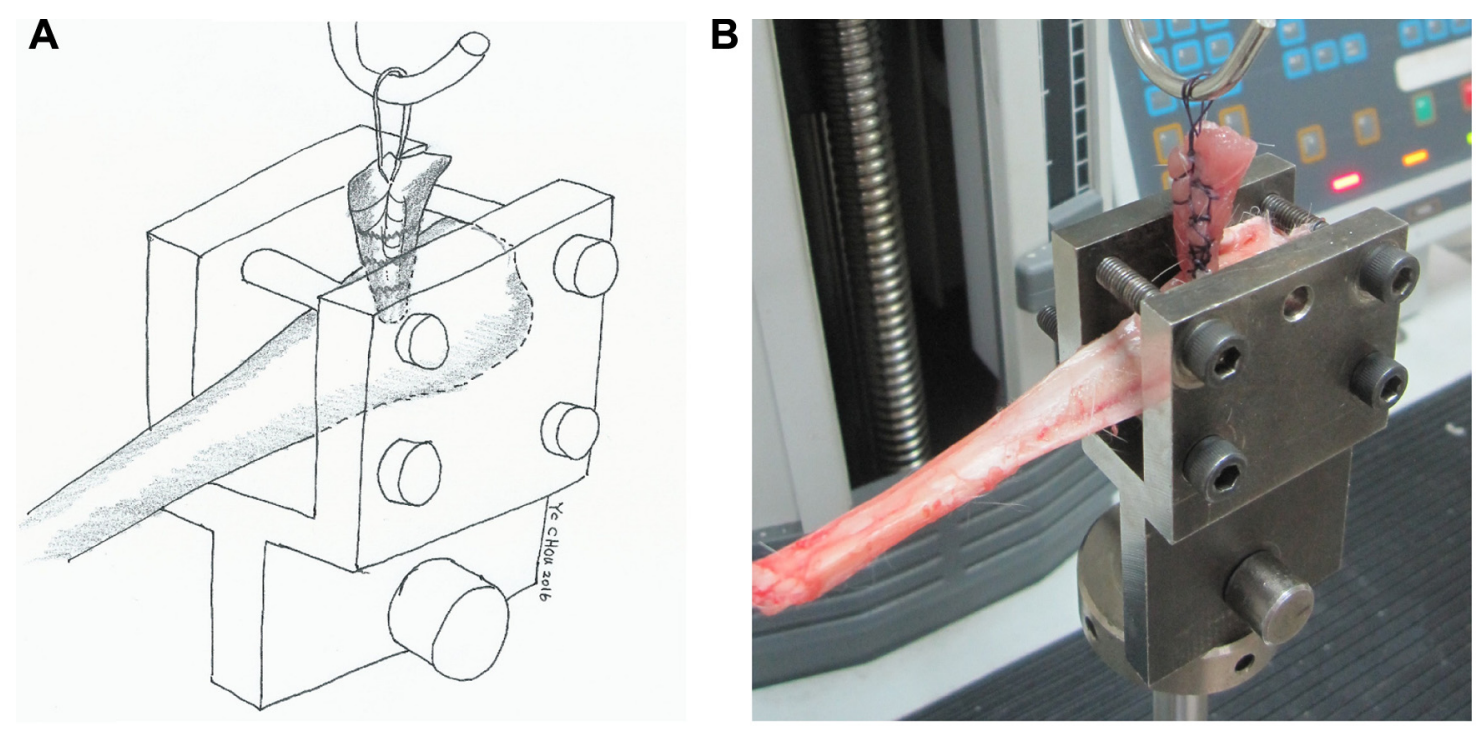

Figure 4 Tendon-bone interface pullout test.

Notes: (A) Illustration and (B) photography show that the tibia was clamped and placed on an MTS machine. The tibia was positioned to allow tensile loading aligned with the long axis of the bone tunnel.

Abbreviation: MTS, material testing system. 


\section{Results}

\section{D printed PLA bolt}

The 3D printed bolt was successfully manufactured using the $3 \mathrm{D}$ printer. The average total time for printing a bolt was $\sim 15$ minutes. After immersion in phosphate-buffered solutions for 3 months, no obvious size or shape change was observed in the PLA bolt. The molecular weight variation of the PLA in the 3D printed bolt was measured by a gel permeation chromatograph. The molecular weight of the PLA study demonstrated significant degradation of the PLA material after immersion in buffered solutions for 3 months (Figure 5).

\section{PLGA/collagen nanofibrous membranes}

A collagen-embedded nanofibrous membrane with five different PLGA/collagen ratios was effectively fabricated by the electrospinning technique. Figure $6 \mathrm{~A}-\mathrm{J}$ shows the SEM images of the nanofibers at a magnification of $\times 4,500$. The average diameters of the nanofibers with different PLGA/ collagen ratios (namely PLGA solution/collagen solution $=40 / 60 \mathrm{v} / \mathrm{v}, 50 / 50 \mathrm{v} / \mathrm{v}, 67 / 33 \mathrm{v} / \mathrm{v}, 80 / 20 \mathrm{v} / \mathrm{v}, 100 / 0 \mathrm{v} / \mathrm{v}$ ) were $214.97 \pm 55.55 \mathrm{~nm}, 594.11 \pm 108.8 \mathrm{~nm}, 826.06 \pm 123.86 \mathrm{~nm}$, $849.86 \pm 139.11 \mathrm{~nm}$, and $913.22 \pm 148.99 \mathrm{~nm}$, respectively (Figure 7). The SEM images clearly indicated that PLGA/ collagen at a 40/60 mixing ratio had a significantly smaller mesh size compared with the other four conditions. This SEM study revealed that the higher the PLGA ratio of the PLGA/ collagen blend solutions, the higher the inconsistency in the nanofiber diameters. In contrast, the higher the collagen ratio of the solutions, the smaller the average diameter of the nanofibers.

Figure $8 \mathrm{~A}-\mathrm{E}$ shows that only the nanofibers with a PLA/collagen ratio of 100/0 exhibited hydrophobic characteristics, whereas the other four nanofibers exhibited hydrophilic characteristics. In addition, the higher the collagen ratio of the PLGA/collagen blend solutions, the greater the hydrophilic nature of the nanofibers, which will in turn promote cell differentiation and tendon reconstruction. The $\mathrm{pH}$ values of the eluted solutions from the PLGA/collagen membranes on various days are shown in Figure 9. All nanofibers with different PLA/collagen ratios maintained their acid-phosphate values between $\mathrm{pH} 7$ and $\mathrm{pH} 8$, which are suitable for cell growth.

After culturing the fibroblasts in DMEM, the OD values considerably increased from day 1 to day 3 for all groups embedded with nanofibers compared with the control group (no nanofibers; $P<0.05$ ). Furthermore, the group with a PLGA/collagen ratio of $67 / 33$ revealed a more significant boost in the OD value $(P<0.01)$. Thus, the nanofibers with a ratio of $67 / 33$ were selected for subsequent animal studies (Figure 10).

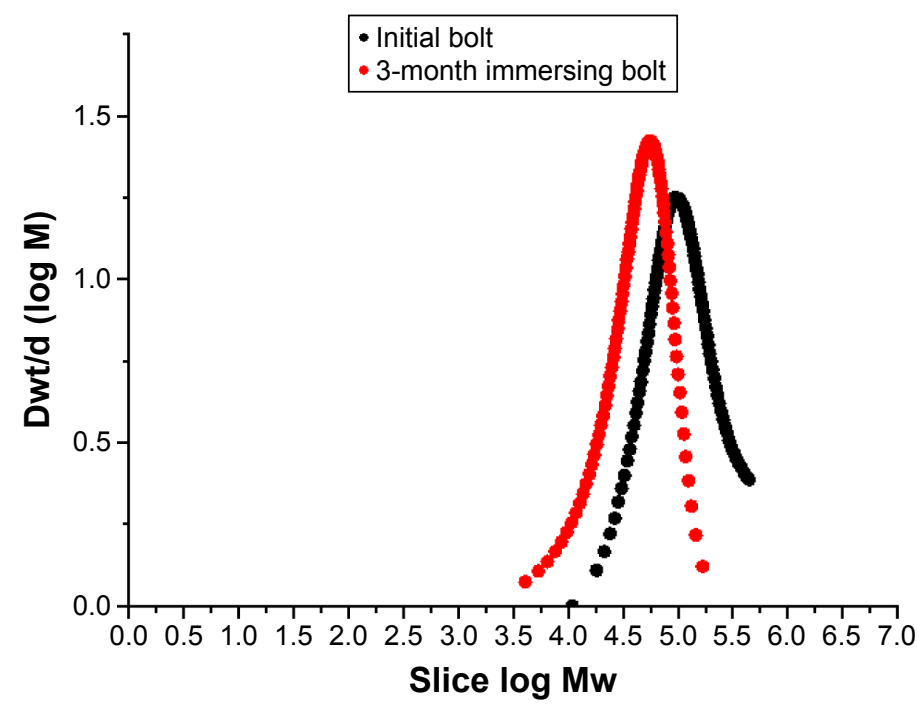

\begin{tabular}{|l|l|l|l|l|}
\hline & $\begin{array}{l}\text { Mn } \\
(\mathrm{Da})\end{array}$ & $\begin{array}{l}\text { Mw } \\
(\mathrm{Da})\end{array}$ & $\begin{array}{l}\text { Mz } \\
(\mathrm{Da})\end{array}$ & $\begin{array}{l}\text { Mz+1 } \\
(\mathrm{Da})\end{array}$ \\
\hline Initial bolt & 75,616 & 129,752 & 202,525 & 271,974 \\
\hline 3-month immersing bolt & 27,319 & 51,274 & 74,738 & 98,718 \\
\hline
\end{tabular}

Figure 5 PLA bolt degradation test by molecular weight measurement

Note: The molecular weight variation of the PLA bolt after immersion in buffer solutions for 3 months.

Abbreviations: PLA, polylactide; Dwt/d, molecular weight distribution; Mn, number average molecular weight; Nw, weight average molecular weight; Mz, Z average molecular weight; $\mathrm{Mz}+\mathrm{I}, \mathrm{Z}+\mathrm{I}$ average molecular weight. 

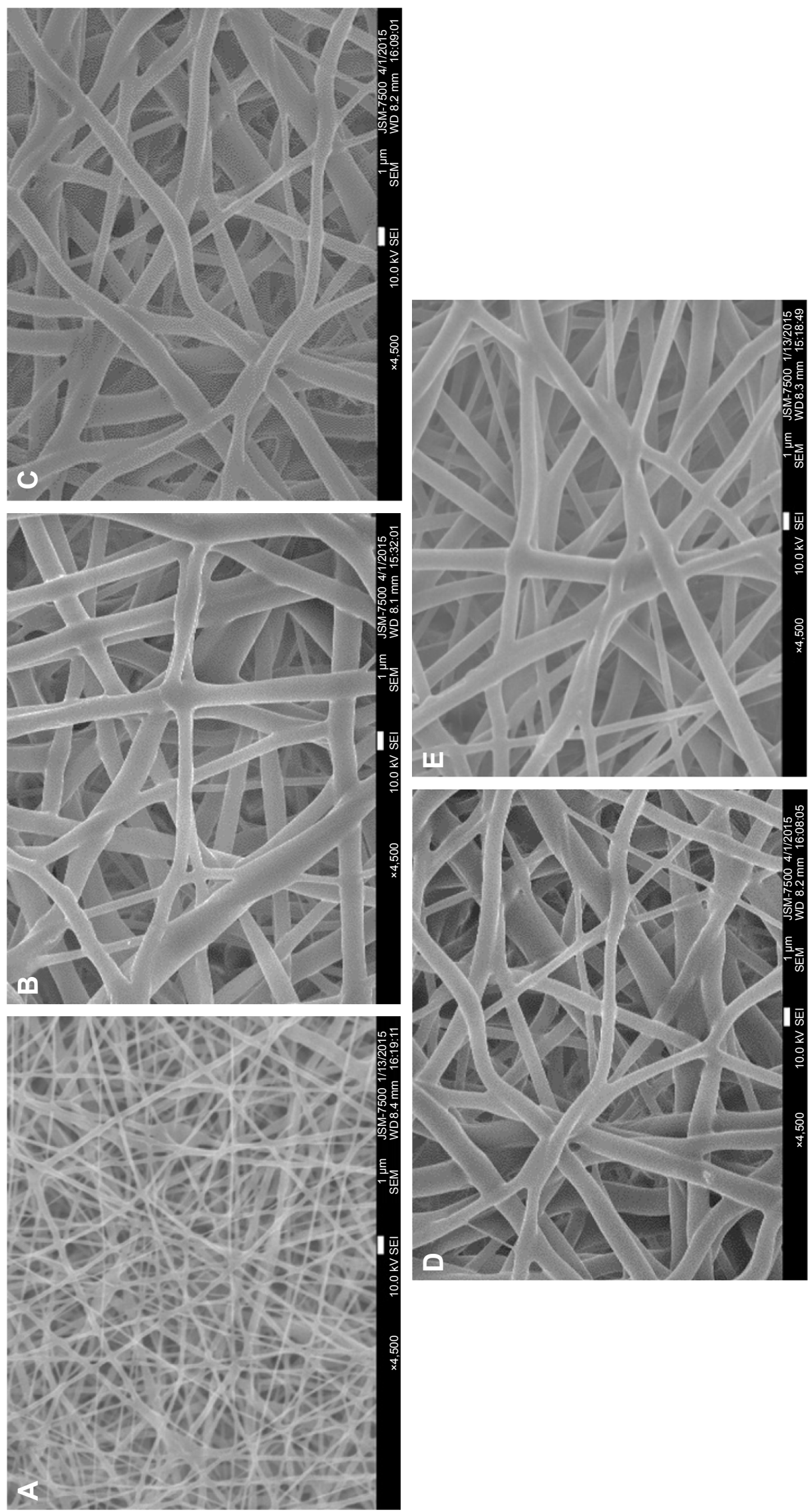

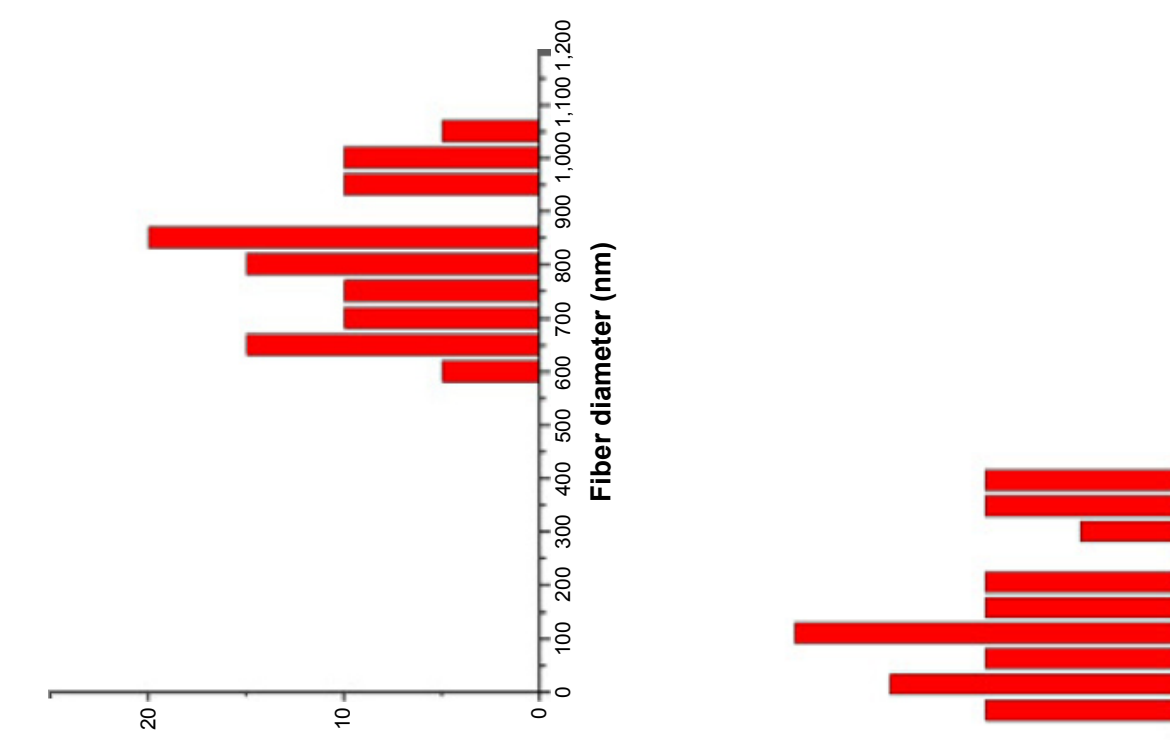

I

(\%) sıəq!! to səqunN
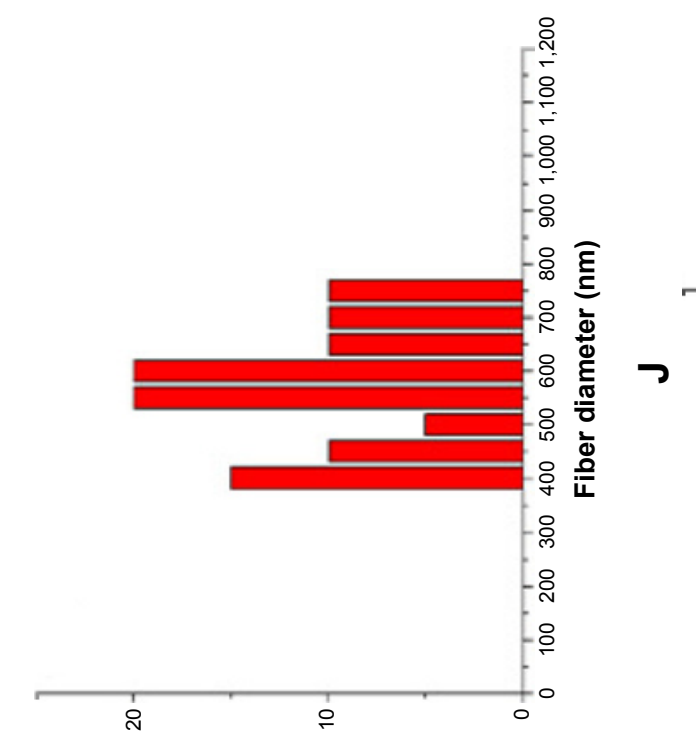

$\boldsymbol{U}$

(\%) sıəq!! to səqunN
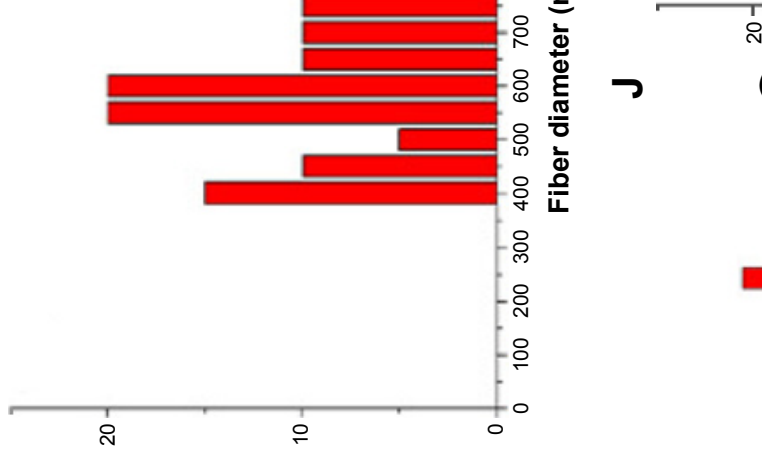

(\%) sıəq! to səqunN
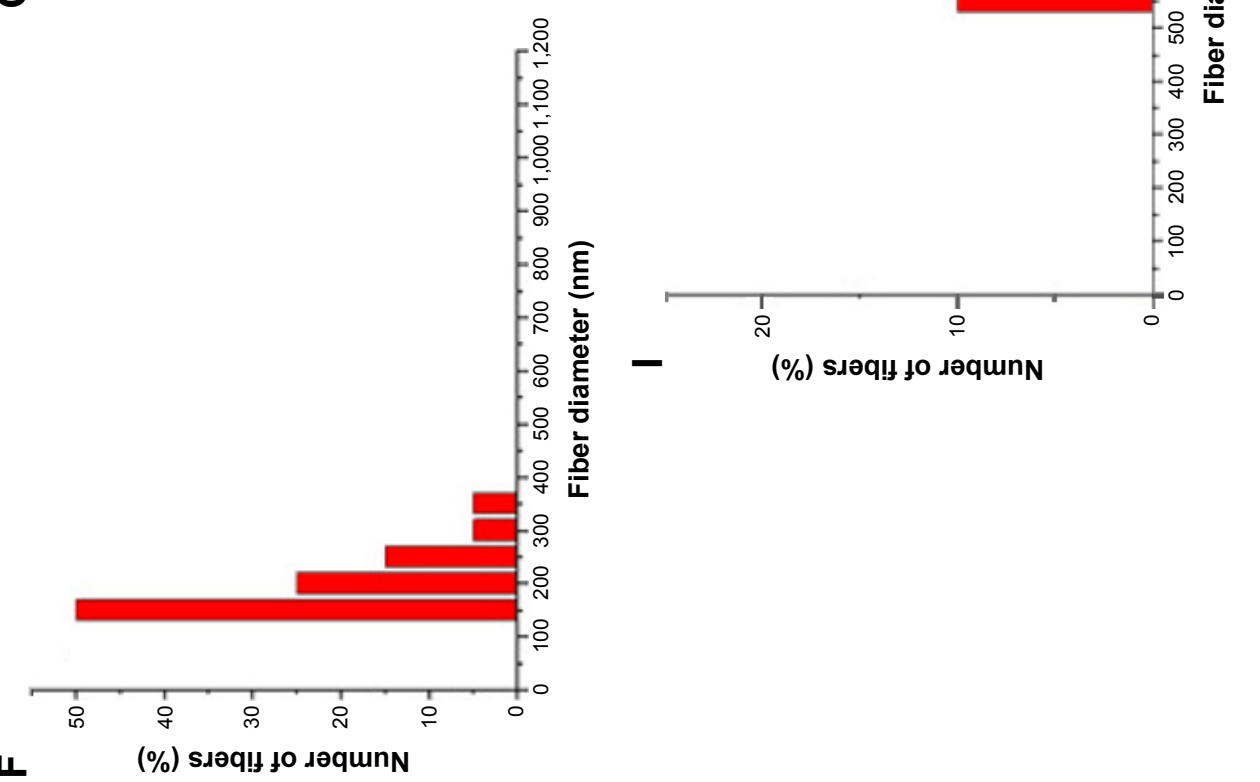

u
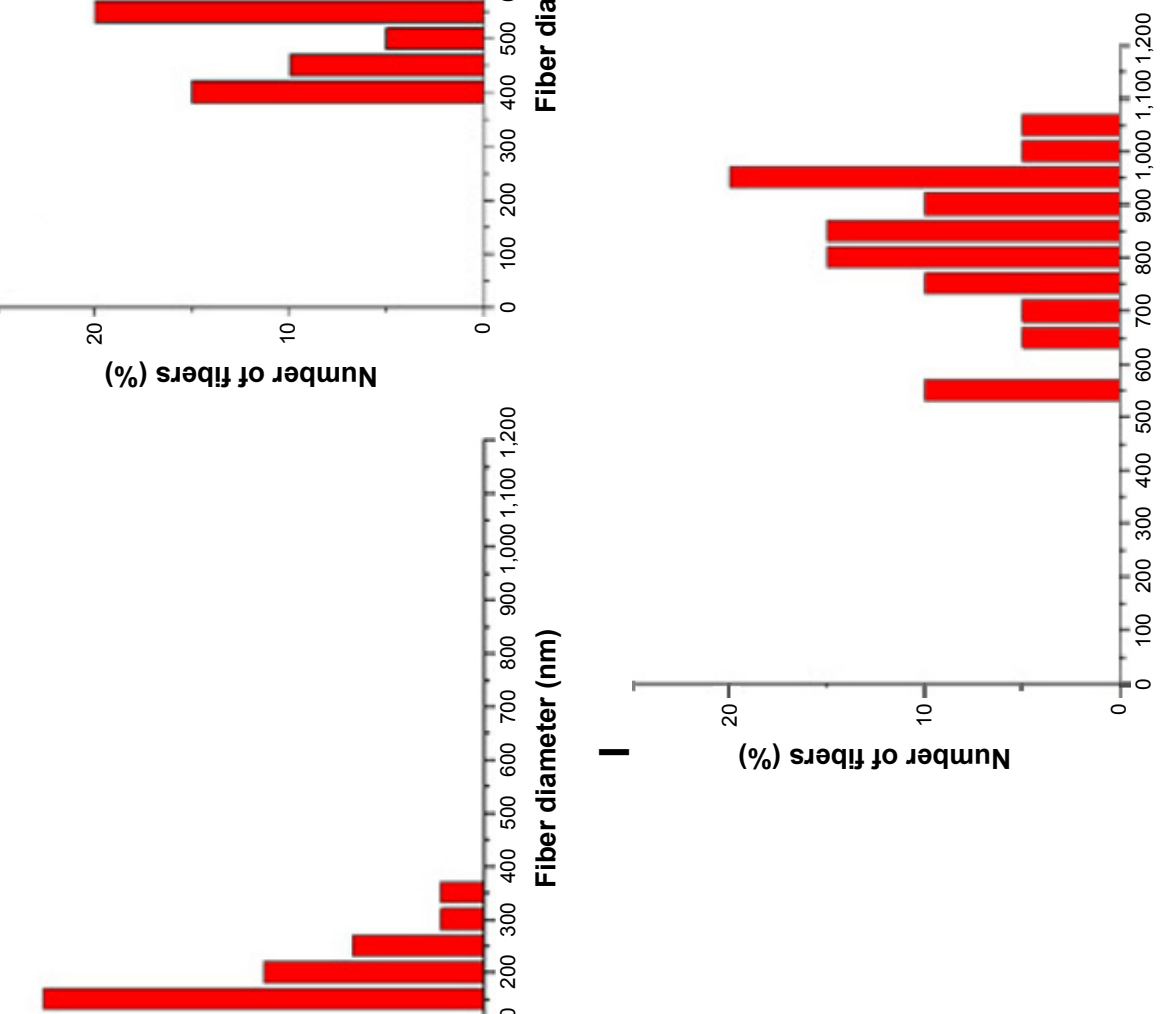

8 हิ

원

过

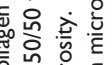

额

을 든

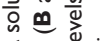

芯文点

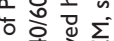

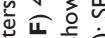

施施

造

to

告 은

号

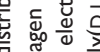

응

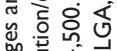

छे 艾文

出仝

뭉

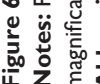




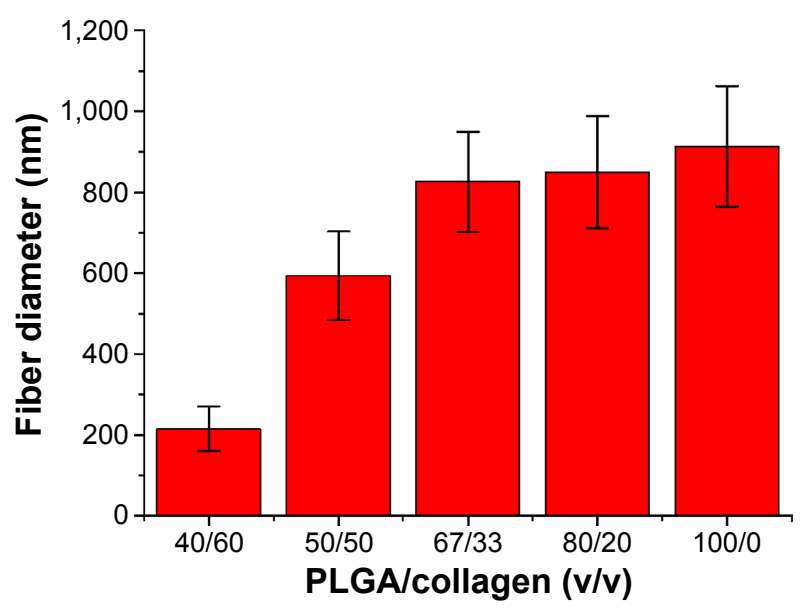

Figure 7 Average diameters of PLGA solution/collagen solutions. Note: PLGA solution/collagen solutions of $40 / 60 \mathrm{v} / \mathrm{v}, 50 / 50 \mathrm{v} / \mathrm{v}, 67 / 33 \mathrm{v} / \mathrm{v}, 80 / 20 \mathrm{v} / \mathrm{v}$, and $100 / 0 \mathrm{v} / \mathrm{v}$ showed average diameters of $214.97 \pm 55.55 \mathrm{~nm}, 594.1 \mathrm{I} \pm 108.8 \mathrm{~nm}$, $826.06 \pm 123.86 \mathrm{~nm}, 849.86 \pm 139.1 \mathrm{Imm}$, and $913.22 \pm \mid 48.99 \mathrm{~nm}$, respectively. Abbreviation: PLGA, poly(D,L-lactide-co-glycolide).

\section{Animal study}

The histological examination of the medial cortical tunnel cross sections revealed progressive new bone formation in the bone tunnel at 8 weeks (Figure 11A). At 16 weeks, the new bone grew around the bolt in a circular manner. There were no tissue overreactions around the bone tunnel and the newly formed bone, which demonstrated the biocompatibility of the 3D printed bolt in the rabbit bone tunnel model (Figure 11B). In 8-week lateral cortical tunnel specimens, most cross sections showed a significant "halo sign" at the tendonbone interface, indicating the bone tunnel enlargement phenomenon. ${ }^{17}$ Only a few fibrous interface layer formations were observed in group A (Figure 12A). There were relatively more fibrovascular interface tissue formations between the tendon and bone junctions in group B (Figure 12B). In 16-week lateral cortical tunnel specimens, the halo portion at the tendon-bone interface significantly decreased in group A and there was progressive mineralization and maturation of the new bone growing into the fibrous interface layer (Figure 12C). In group B, progressive fiber-bone anchorage, new bone maturation, and organization between the tendon and bone lining developed (Figure 12D).

For the tendon-bone interface strength test at 8 weeks, $75 \%$ (nine of 12 ) of the tendons in group A were pulled out from the bone tunnel at the failure point, while the other $25 \%$ (three of 12 ) of the tendons failed by rupture of the mid-substance of the tendon or at the tendon-bone interface. On the other hand, $58 \%$ (seven of 12) of the tendons in group B were noted to be pulled out from the bone tunnel and $42 \%$ (seven of 12) failed by rupture of the tendons. There was no significant difference between the maximal failure loads of these two groups at this time interval. At 16 weeks, 33\% (four of 12) of the tendons were pulled out from the bone tunnel and $67 \%$ (eight of 12 ) ruptured at the tendon. At the same time, only $18 \%$ (two of 12 ) of the tendons in group B were pulled out from the bone tunnel and $83 \%$ (ten of 12) broke at the tendon portion. The average maximal failure load of group B was 41.4 N (37.4-44.5 N), which is significantly greater than that of group A $(28.3 \mathrm{~N} ; 26.1-30.5 \mathrm{~N})$ at 16 weeks (Figure 13).

\section{Discussion}

Tendon injuries are common in orthopedic clinical practice and cause substantial morbidity in sports and routine daily activities. Tendon-bone healing is vital for the ultimate success of various surgical procedures performed to repair injured tendons. ${ }^{3,21,22}$ Reports indicated that enveloping the tendon graft with periosteum may improve osseous ingrowth and healing at the tendon-bone interface because periosteum has a unique population of mesenchymal stem cells to form various connective tissues and induce new bone formation. ${ }^{8,9,23,24}$ Although enveloping the grafts with periosteum appears to be a promising approach, clinical evidence supporting its use in humans to augment tendon-bone healing is still lacking, and donor site morbidity is an inevitable problem. ${ }^{9,24,25}$ Therefore, biodegradable scaffolds or biomimetic patches have also been introduced for enveloping
A

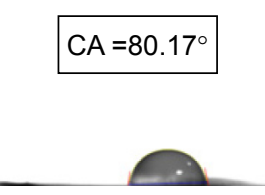

$40 / 60 \mathrm{v} / \mathrm{v}$
B

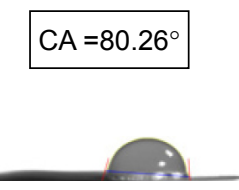

$50 / 50 \mathrm{v} / \mathrm{v}$
C

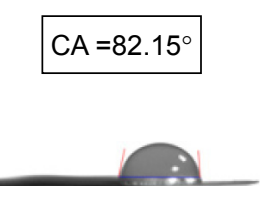

$67 / 33 \mathrm{v} / \mathrm{v}$
D

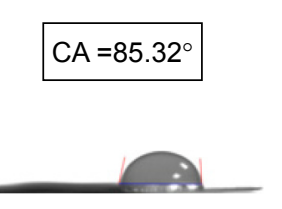

$80 / 20 \mathrm{v} / \mathrm{v}$
E $\mathrm{CA}=100.61^{\circ}$

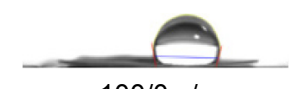

$100 / 0 \mathrm{v} / \mathrm{v}$

Figure 8 Water CAs of PLGA solution/collagen solutions.

Notes: Nanofibrous membranes with different PLGA/collagen ratios of (A) 40/60 v/v, (B) 50/50 v/v, (C) 67/33 v/v, (D) 80/20 v/v, and (E) $100 / 0 \mathrm{v} / \mathrm{v}$ revealed that only the nanofiber with a PLGA/collagen ratio of $100 / 0 \mathrm{v} / \mathrm{v}$ was hydrophobic, while the other four nanofibers were hydrophilic. Abbreviations: CA, contact angle; PLGA, poly(D,L-lactide-co-glycolide). 


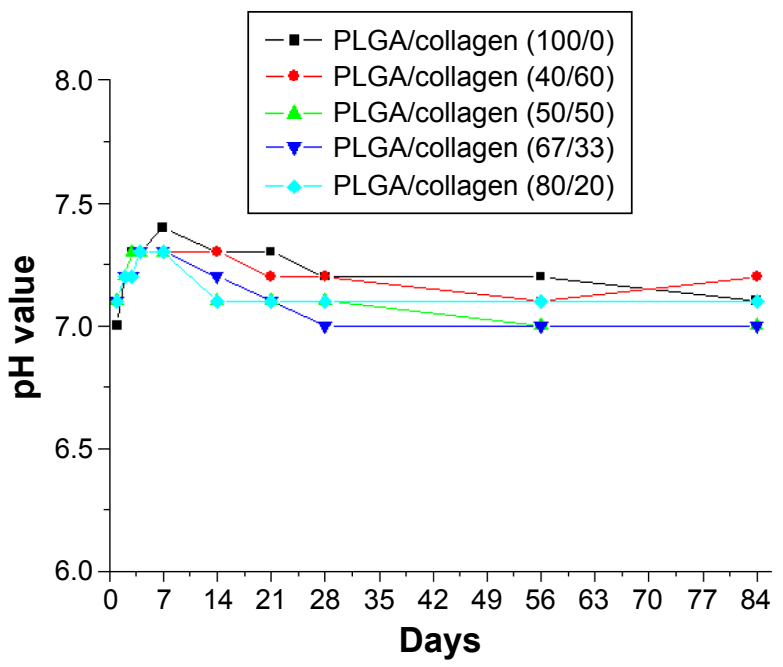

Figure $9 \mathrm{pH}$ values of eluted solutions from the PLGA/collagen membranes on various days.

Note: All nanofibers with different PLGA/collagen ratios maintained their acidphosphate values between $\mathrm{pH} 7$ and $\mathrm{pH}$ 8, which are suitable for cell growth. Abbreviation: PLGA, poly(D,L-lactide-co-glycolide).

the tendon graft to promote tendon-bone healing. ${ }^{18,26}$ In the current study, we selected collagen and PLGA to build up a nanofibrous membrane and act as a biomimetic periosteum to enhance tissue invasion and cell attachments in the tendonbone tunnel interface.

PLGA is one of the biodegradable materials that has been approved by the US Food and Drug Administration for medical use and has been widely studied as a therapeutic delivery vehicle owing to its biocompatibility and biodegradability. ${ }^{27,28}$ After being introduced into the body, PLGA induces only a minimal inflammatory response and biodegrades through the hydrolysis of its ester linkages to

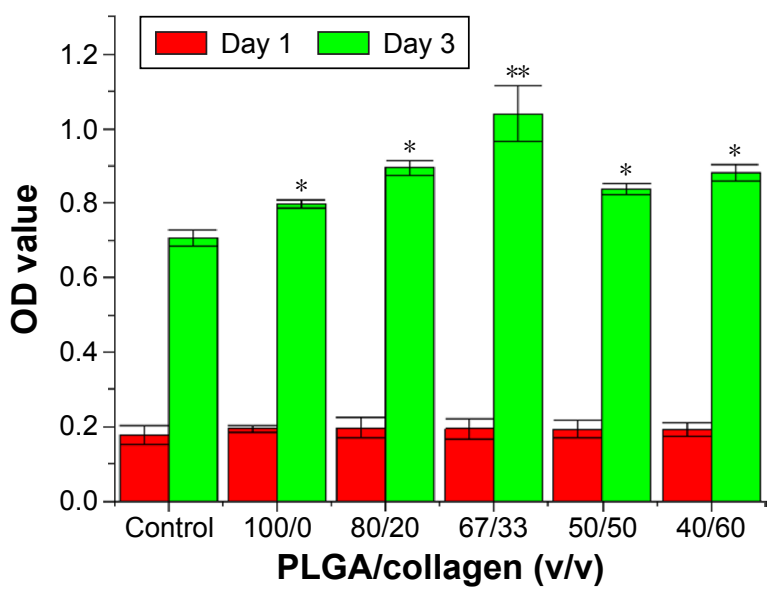

Figure 10 Results of cytotoxicity testing of the nanofibrous membrane. Notes: The OD value increased from day I to day 3 in all nanofiber-embedded groups compared with the control group. The group with a PLGA/collagen ratio of $67 / 33$ revealed a more significant boost in the OD value. $* P<0.05 ; * * P<0.01$. Abbreviations: OD, optical density; PLGA, poly(D,L-lactide-co-glycolide). yield biocompatible lactic and glycolic acids. ${ }^{29,30}$ The in vitro tests in this study showed that the PLGA/collagenblended nanofibrous membrane is a hydrophilic, acid-base stable, and biocompatible polymer suitable for biological applications.

Collagen is a natural extracellular matrix component of many tissues, such as skin, bone, tendon, ligament, and other connective tissues. Among the collagen isotypes, type I is the principal structural and functional protein. ${ }^{31,32}$ The fibrillar structure of type I collagen has long been known to be important for cell attachment, proliferation, and differentiated function in tissue culture. ${ }^{33}$ In native tissue, type I collagen fibrils have diameters ranging from $50 \mathrm{~nm}$ to $500 \mathrm{~nm}$ and are quite uniform in size. The SEM results of this study showed that all PLGA/collagen nanofibrous membranes with five different compositions, and with average diameters ranging from $200 \mathrm{~nm}$ to $1,100 \mathrm{~nm}$, have porous ultrastructures of $<1,200 \mathrm{~nm}$, which were demonstrated earlier to permit tissue invasion and cell attachment. . $^{31,34}$

In this study, we designed and developed a truncated cone-shaped PLA-anchoring bolt to act as a circumferential press-fit fixation of the tendon at the medial cortical site. The in vitro characterization in this study demonstrated degradation of the molecular weight of the 3D printed bolts at 3 months. It has been reported that high molecular weight PLA can take between 2 years and 5.6 years for total resorption in vivo. Although the polymer is known to lose its strength in $\sim 6$ months when hydrolyzed, no significant changes in mass will occur for a very long time. ${ }^{14,15}$ The histological examinations demonstrated adequate biocompatibility of the PLA bolt on the medial cortex with progressive bone ingrowth and without tissue overreaction. By combining the use of a collagen nanofibrous membrane on the lateral cortex with a PLA bolt on the medial cortex, the tendon graft in group B demonstrated adequate stabilization without tunnel enlargement. In contrast, tendon grafts in group A with a PLA bolt on the medial cortex and without nanofibers on the lateral cortex showed halo sign formation, which represented tunnel enlargement on the lateral cortex at the 8-week histological assessment.

The tendon pullout test at the postoperative 8-week assessment showed no significant difference between these two groups owing to adequate press-fit fixation by bolt anchoring at the medial cortex. However, at the 16-week assessment, the different tendon pullout strengths between these two groups revealed that the PLGA/collagen nanofibrous membrane triggered more tendon-bone integrations on the lateral cortex. At the pullout test, the failure point 


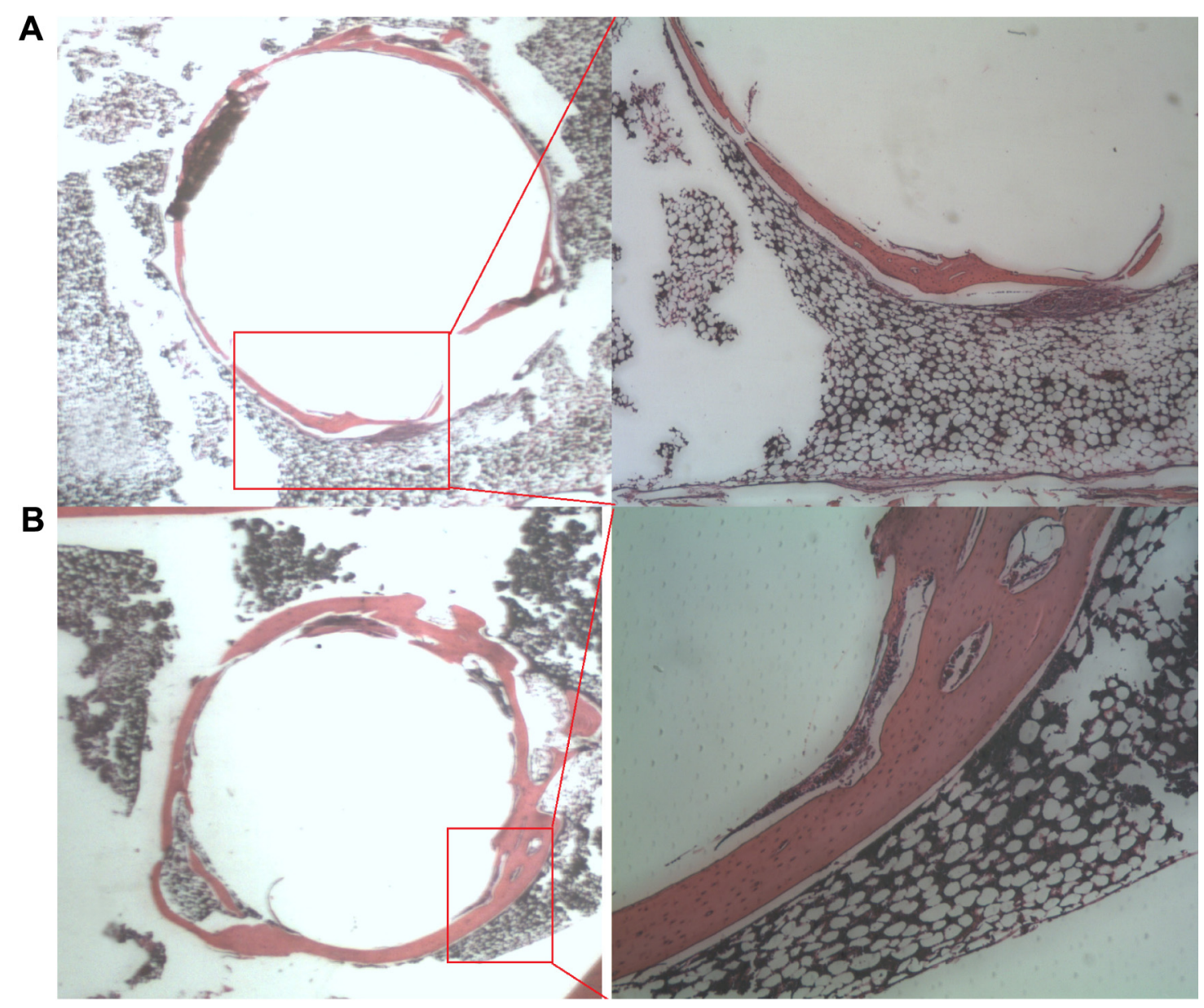

Figure II Histological findings on the medial cortex of the bolt anchoring site.

Notes: (A) Histology of the tendon-bone interface on the medial cortex at 8 weeks (H\&E, original magnification $\times 60$, amplified magnification $\times 150)$ showed mild bone ingrowth around the bolt. (B) Histology of the tendon-bone interface at 16 weeks (H\&E, original magnification $\times 60$, amplified magnification $\times 200)$ demonstrated more bone ingrowth around the bolt.

Abbreviation: H\&E, hematoxylin and eosin.

represented the key weakness structure of the healing tendon graft. These failures by tendon pullout from the tunnel represented a decreased press-fit effect by the PLA bolt with inadequate tendon-bone integration. The ruptures on the mid-substance of the tendon represented an adequate press-fit effect by the PLA bolt and incorporation on the tendon-bone interface, such that the strength of tendon-bone integration exceeded that of the tendon itself. Thus, the break point was on the mid-substance of the tendon rather than on the tendon-bone interface.

The results of this study demonstrated that the composite polymers of the PLA bolt and PLGA/collagen nanofibrous membrane can effectively promote outcomes of tendon reconstruction in this rabbit model. However, the relatively restrictive sample size of four for the histological assessments and eight for the tendon pullout tests per study arm is a limitation of this study. While most previous studies have used a study population of at least 15 per group, ${ }^{2,35}$ our study insisted on adequate animal care. We did not perform the index procedure and in-contrast operation on both legs of a rabbit. In addition, we did not perform an animal study of in vivo comparisons for all five PLGA/collagen compositions owing to the limitation in the number of rabbits. We only selected the most bioeffective composition of a PLGA/ collagen ratio of $67 / 33$ for the in vivo animal studies. All these limitations will be topics of future study.

\section{Conclusion}

Successful tendon reconstruction can be challenging because of the limited regeneration capacity of the tendon-bone interface and decisive requirement of tendon-bone anchoring to avoid the tunnel enlargement phenomenon. Our design of the PLA bolts provides durable stabilization of the tendon graft for tendon-bone healing. The PLGA/collagen-blended nanofibrous membrane also enhances tendon-bone healing by increasing tissue invasion and cell attachment. 
A

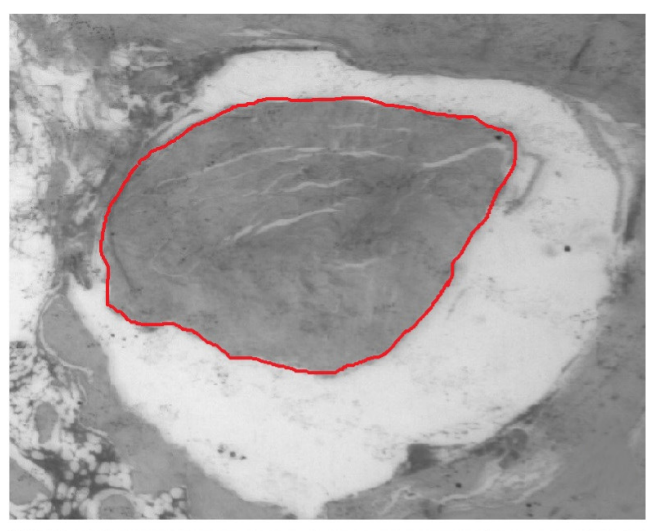

B

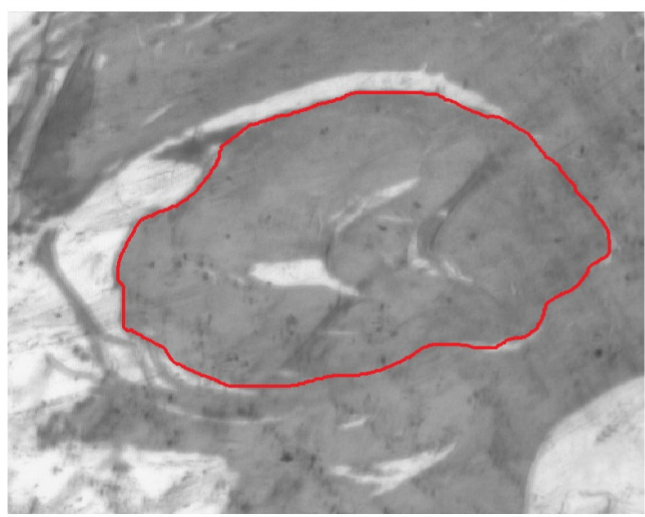

C

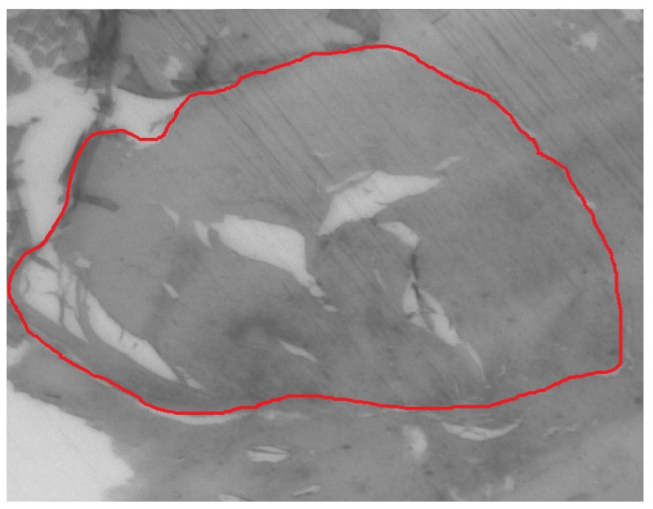

D

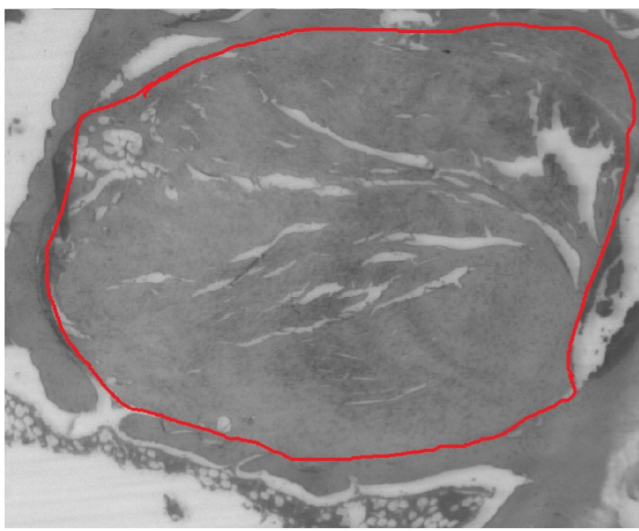

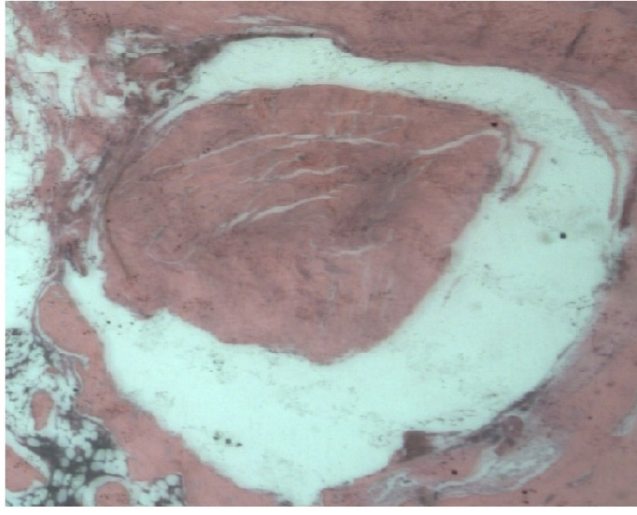
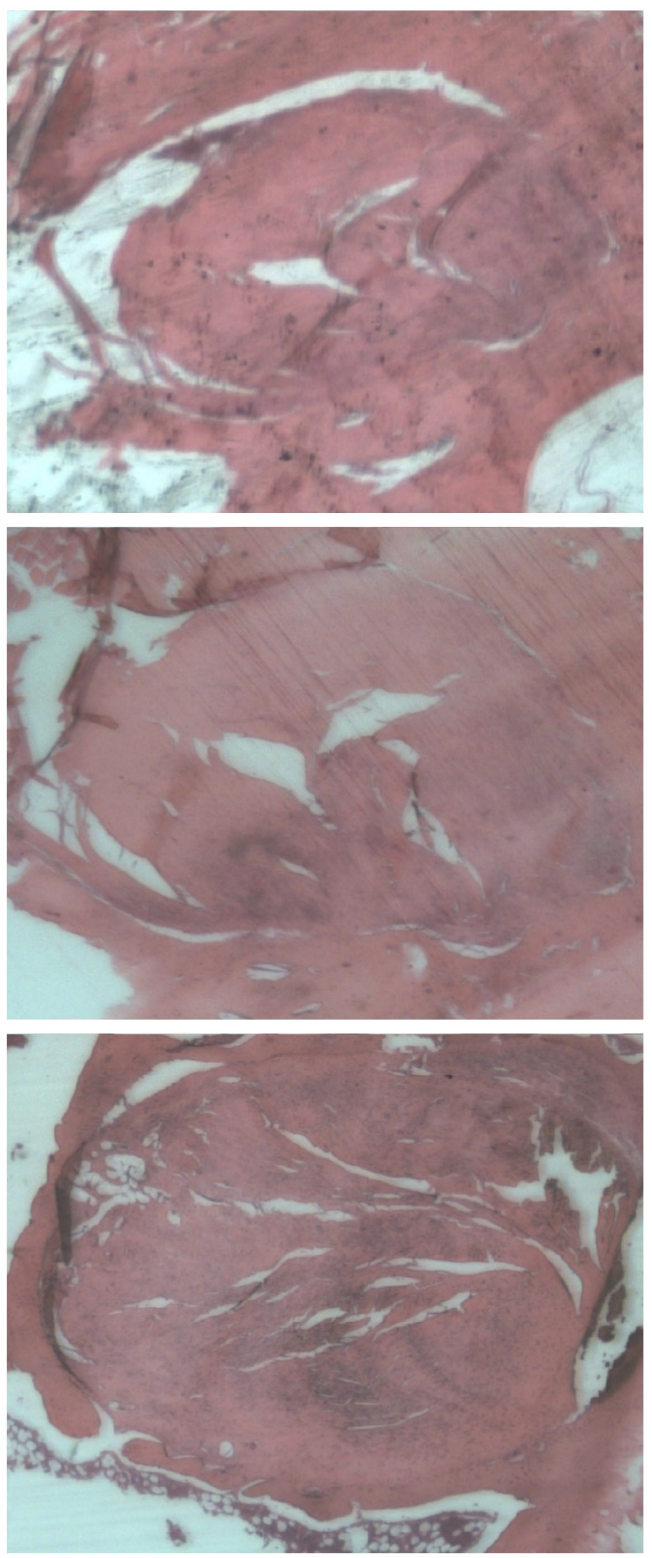

Figure 12 The comparisons of tendon-marginal illustrations with a red circle indicating the tendon margin and histological findings with H\&E stain on the tendon-bone interfaces between group $A$ (without nanofibers) and group $B$ (with nanofibers).

Notes: (A) Histology of a tendon-bone interface on the lateral cortex in group $A$ at 8 weeks (H\&E, magnification $\times 60)$ showed the tunnel enlargement phenomenon. (B) A tendonbone interface on the lateral cortex in group $B$ at 8 weeks showed more fibrovascular interface tissue formations between the tendon and bone. (C) $A$ tendon-bone interface on the lateral cortex in group $A$ at 16 weeks revealed progressive mineralization and maturation of the new bone growing into the interface fibrous layer. (D) A tendon-bone interface on the lateral cortex in group B at 16 weeks demonstrated progressive fiber-bone anchorage, new bone maturation, and organization between the tendon and bone.

Abbreviation: H\&E, hematoxylin and eosin. 


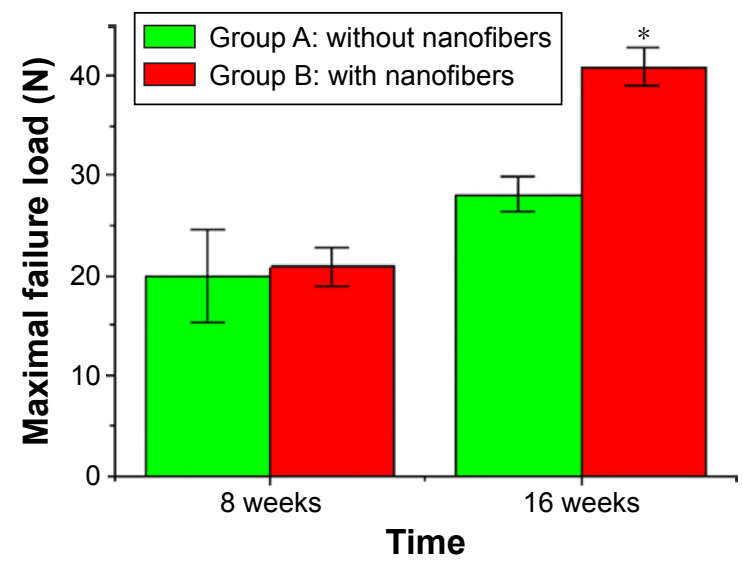

Figure 13 The comparisons of average maximal failure loads for the tendon pullout test between group $A$ and group $B$.

Notes: The average maximal failure load of group B was $41.4 \mathrm{~N}(37.4-44.5 \mathrm{~N})$, which was significantly greater than that of group $A(28.3 \mathrm{~N} ; 26 . \mathrm{I}-30.5 \mathrm{~N})$ at 16 weeks. $* P<0.05$.

\section{Acknowledgment}

The authors thank Chang Gung Memorial Hospital (contract number CMRPD2E0041) for financially supporting this research.

\section{Disclosure}

The authors report no conflicts of interest in this work.

\section{References}

1. Ait Si Selmi T, Fithian D, Neyret P. The evolution of osteoarthritis in 103 patients with ACL reconstruction at 17 years follow-up. Knee. 2006;3(5):353-358.

2. Pinczewski LA, Lyman J, Salmon LJ, Russell VJ, Roe J, Linklater J. A 10-year comparison of anterior cruciate ligament reconstructions with hamstring tendon and patellar tendon autograft: a controlled, prospective trial. Am J Sports Med. 2007;35(4):564-574.

3. Gulotta LV, Kovacevic D, Ying L, Ehteshami JR, Montgomery $\mathrm{S}$, Rodeo SA. Augmentation of tendon-to-bone healing with a magnesium-based bone adhesive. Am J Sports Med. 2008;36(7): $1290-1297$.

4. Newsham-West R, Nicholson H, Walton M, Milburn P. Long-term morphology of a healing bone-tendon interface: a histological observation in the sheep model. J Anat. 2007;210(3):318-327.

5. Rodeo SA, Potter HG, Kawamura S, Turner AS, Kim HJ, Atkinson BL. Biologic augmentation of rotator cuff tendon-healing with use of a mixture of osteoinductive growth factors. J Bone Joint Surg Am. 2007; 89(11):2485-2497.

6. Alsousou J, Ali A, Willett K, Harrison P. The role of platelet-rich plasma in tissue regeneration. Platelets. 2013;24(3):173-182.

7. Mazzocca AD, McCarthy MB, Chowaniec DM, et al. The positive effects of different platelet rich plasma methods on human muscle, bone, and tendon cells. Am J Sports Med. 2012;40(8):1742-1749.

8. Chang $\mathrm{CH}$, Chen $\mathrm{CH}, \mathrm{Su} \mathrm{CY}$, Liu HT, Yu CM. Rotator cuff repair with periosteum for enhancing tendon-bone healing: a biomechanical and histological study in rabbits. Knee Surg Sports Traumatol Arthrosc. 2009; 17(12):1447-1453.

9. Karaoglu S, Celik C, Korkusuz P. The effects of bone marrow or periosteum on tendon-to-bone tunnel healing in a rabbit model. Knee Surg Sports Traumatol Arthrosc. 2009;17(2):170-178.
10. Weiler A, Hoffmann RF, Bail HJ, Rehm O, Sudkamp NP. Tendon healing in a bone tunnel. Part II: histologic analysis after biodegradable interference fit fixation in a model of anterior cruciate ligament reconstruction in sheep. Arthroscopy. 2002;18(2):124-135.

11. Hertel P, Behrend H, Cierpinski T, Musahl V, Widjaja G. ACL reconstruction using bone-patellar tendon-bone press-fit fixation: 10-year clinical results. Knee Surg Sports Traumatol Arthrosc. 2005;13(4): 248-255.

12. Kaushik NK, Kaushik N, Pardeshi S, Sharma JG, Lee SH, Choi EH. Biomedical and clinical importance of mussel-inspired polymers and materials. Mar Drugs. 2015;13(11):6792-6817.

13. Yamada S, Obata A, Maeda H, Ota Y, Kasuga T. Development of magnesium and siloxane-containing vaterite and its composite materials for bone regeneration. Front Bioeng Biotechnol. 2015;3:195.

14. Pinese C, Leroy A, Nottelet B, Gagnieu C, Coudane J, Garric X. Rolled knitted scaffolds based on PLA-pluronic copolymers for anterior cruciate ligament reinforcement: a step by step conception. J Biomed Mater Res B Appl Biomater. Epub 2016 Jan 5.

15. Zhu X, Zhong T, Huang R, Wan A. Preparation of hydrophilic poly(lactic acid) tissue engineering scaffold via (PLA)-(PLA-bPEG)-(PEG) solution casting and thermal-induced surface structural transformation. J Biomater Sci Polym Ed. 2015;26(17):1286-1296.

16. Spalazzi JP, Dagher E, Doty SB, Guo XE, Rodeo SA, Lu HH. In vivo evaluation of a multiphased scaffold designed for orthopaedic interface tissue engineering and soft tissue-to-bone integration. J Biomed Mater Res A. 2008;86(1):1-12.

17. Park S, Kang YJ, Majd S. Patterned materials: a review of patterned organic bioelectronic materials and their biomedical applications. Adv Mater. 2015;27(46):7486.

18. Giza E, Frizzell L, Farac R, Williams J, Kim S. Augmented tendon Achilles repair using a tissue reinforcement scaffold: a biomechanical study. Foot Ankle Int. 2011;32(5):S545-S549.

19. De Santis R, Russo A, Gloria A, et al. Towards the design of 3D fiber-deposited poly( $\varepsilon$-caprolactone)/iron-doped hydroxyapatite nanocomposite magnetic scaffolds for bone regeneration. J Biomed Nanotechnol. 2015;11:1236-1246.

20. Liu SJ, Chiang FJ, Hsiao CY, Kau YC, Liu KS. Fabrication of balloonexpandable self-lock drug-eluting polycaprolactone stents using microinjection molding and spray coating techniques. Ann Biomed Eng. 2010;38(10):3185-3194.

21. Paessler HH, Mastrokalos DS. Anterior cruciate ligament reconstruction using semitendinosus and gracilis tendons, bone patellar tendon, or quadriceps tendon-graft with press-fit fixation without hardware: a new and innovative procedure. Orthop Clin North Am. 2003;34(1):49-64.

22. Yun YR, Won JE, Jeon E, et al. Fibroblast growth factors: biology, function, and application for tissue regeneration. J Tissue Eng. 2010; 2010(7):218142.

23. Chen $\mathrm{CH}$, Chen WJ, Shih $\mathrm{CH}$. Enveloping of periosteum on the hamstring tendon graft in anterior cruciate ligament reconstruction. Arthroscopy. 2002;18(5):27E.

24. Ferretti C, Borsari V, Falconi M, et al. Human periosteum-derived stem cells for tissue engineering applications: the role of VEGF. Stem Cell Rev. 2012;8(3):882-890.

25. Li H, Jiang J, Wu Y, Chen S. Potential mechanisms of a periosteum patch as an effective and favourable approach to enhance tendon-bone healing in the human body. Int Orthop. 2012;36(3):665-669.

26. Shi Z, Xu T, Yuan Y, et al. A new absorbable synthetic substitute with biomimetic design for dural tissue repair. Artif Organs. 2016;40(4): 403-413.

27. Goffin AL, Raquez JM, Duquesne E, et al. From interfacial ring-opening polymerization to melt processing of cellulose nanowhisker-filled polylactide-based nanocomposites. Biomacromolecules. 2011;12: 2456-2465.

28. Zhu J, Yang F, He F, Tian X, Tang S, Chen X. A tubular gelatin scaffold capable of the time-dependent controlled release of epidermal growth factor and mitomycin C. Colloids Surf B Biointerfaces. 2015;135: 416-424. 
29. Mohammadi-Samani S, Taghipour B. PLGA micro and nanoparticles in delivery of peptides and proteins; problems and approaches. Pharm Dev Technol. 2015;20(4):385-393.

30. Pandita D, Kumar S, Lather V. Hybrid poly(lactic-co-glycolic acid) nanoparticles: design and delivery prospectives. Drug Discov Today. 2015;20(1):95-104.

31. Kuzmenko V, Sämfors S, Hägg D, Gatenholm P. Universal method for protein bioconjugation with nanocellulose scaffolds for increased cell adhesion. Mater Sci Eng C Mater Biol Appl. 2013;33(8):4599-4607.

32. Hee CK, Dines JS, Dines DM, et al. Augmentation of a rotator cuff suture repair using rhPDGF-BB and a type I bovine collagen matrix in an ovine model. Am J Sports Med. 2011;39(8):1630-1639.
33. Mazzocca AD, McCarthy MB, Arciero C, et al. Tendon and bone responses to a collagen-coated suture material. J Shoulder Elbow Surg. 2007;16(5):S222-S230.

34. Schuldt C, Karl A, Körber N, et al. Dose-dependent collagen crosslinking of rabbit scleral tissue by blue light and riboflavin treatment probed by dynamic shear rheology. Acta Ophthalmol. 2015;93(5): e328-e336.

35. Boszotta H. Arthroscopic anterior cruciate ligament reconstruction using a patellar tendon graft in press-fit technique: surgical technique and follow-up. Arthroscopy. 1997;13(3):332-339.

\section{Publish your work in this journal}

The International Journal of Nanomedicine is an international, peerreviewed journal focusing on the application of nanotechnology in diagnostics, therapeutics, and drug delivery systems throughout the biomedical field. This journal is indexed on PubMed Central, MedLine, CAS, SciSearch ${ }^{\circledR}$, Current Contents ${ }^{\circledR} /$ Clinical Medicine,
Journal Citation Reports/Science Edition, EMBase, Scopus and the Elsevier Bibliographic databases. The manuscript management system is completely online and includes a very quick and fair peer-review system, which is all easy to use. Visit http://www.dovepress.com/ testimonials.php to read real quotes from published authors. 